\title{
Modified Particle Swarm Optimization Applied to Integrated Demand Response and DG Resources Scheduling
}

\author{
Pedro Faria, João Soares, Zita Vale, Hugo Morais and Tiago Sousa
}

\begin{abstract}
The elastic behavior of the demand consumption jointly used with other available resources such as distributed generation (DG) can play a crucial role for the success of smart grids. The intensive use of Distributed Energy Resources (DER) and the technical and contractual constraints result in large-scale non linear optimization problems that require computational intelligence methods to be solved.

This paper proposes a Particle Swarm Optimization (PSO) based methodology to support the minimization of the operation costs of a virtual power player that manages the resources in a distribution network and the network itself. Resources include the DER available in the considered time period and the energy that can be bought from external energy suppliers. Network constraints are considered. The proposed approach uses Gaussian mutation of the strategic parameters and contextual self-parameterization of the maximum and minimum particle velocities.

The case study considers a real 937 bus distribution network, with 20310 consumers and 548 distributed generators. The obtained solutions are compared with a deterministic approach and with PSO without mutation and Evolutionary PSO, both using self-parameterization.
\end{abstract}

Index Terms-Demand response, energy resource management, particle swarm optimization, virtual power player.

\section{NOMENCLATURE}

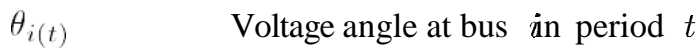

$\theta_{i}^{\max } \quad$ Maximum voltage angle at bus $i$

$\theta_{i}^{\min }$

$c_{A(\mathrm{DG}, t)}$

Minimum voltage angle at bus

Fixed cost coefficient of DG unit in period $t$

$c_{B(\mathrm{DG}, t)}$
$c_{C(\mathrm{DG}, t)}$

$c_{\mathrm{Ch}(S, t)}$

$c_{\operatorname{Dch}(S, t)}$

$c_{\text {Red_A } A, t)}$

$c_{\text {Red_B }}(L, t)$

$c_{\text {Red_C }(L, t)}$

$c_{\mathrm{EAP}(\mathrm{DG}, t)}$

$c_{\mathrm{NSD}(L, t)}$

$B_{i j}$

$G_{i j}$

$N_{B}$

$N_{\text {DG }}$

$N_{\text {DG }}^{i}$

$N_{k}$

$N_{L}$

$N_{L}^{i}$

$N_{S}$

$N_{S}^{i}$

$N_{\text {SP }}^{i}$

$P_{\mathrm{Ch}(S . t)}$

$P_{\text {rhas }}^{i}$

$P_{\text {DG(DG }, t)}$

$P_{\text {DG }}^{i}$ DG,$\left.t\right)$
Quadratic cost coefficient of DG unit in period $t$

Charge cost of storage $S$ in period $t$ Discharge cost of storage $S$ in period $t$

RedAprogram cost, for loadin/period $\quad t$

RedBprogram cost, for loadinLperiod $\quad t$ RedCprogram cost, for loadinLperiod $t$

Excess available power cost coefficient of DG unit in period $t$

Non-supplied demand cost of load $L$ in period $t$

Imaginary part of the element in admittance matrix corresponding to the row and $j$ column

Real part of the element in admittance matrix corresponding to the $i$ row and $j$ column

Total number of buses

Total number of distributed generators

Total number of DG units for the bus $i$

Total number of lines

Total number of loads

Total number of $L$ loads for the bus $i$

Total number of storage units

Total number of $S$ storage units for the bus $i$

Total number of external suppliers

Total number of SP external suppliers for the bus $i$

Active power charge of storage $S$ in period $t$

Active power charge of storage $S$ at bus $i$ in period $t$

Active power generation of distributed generation unit DG in period $t$

Active power generation of distributed generation unit DG at bus in period $t$ 
$P_{\text {DGMax(DG,t) }}$ Maximum active power generation of distributed generator unit DG in period $t$

$P_{\text {DGMin(DG,t) }}$ Minimum active power generation of distributed generator unit DG in period $t$

$P_{\text {Dch(S,t) }} \quad$ Active power discharge of storage $S$ in period $t$

$P_{R E D_{-} A(L, t)}$ RedAactive power reduction, for load $L$ in period $t$

$P_{R E D_{-} A(L, t)}^{i} \operatorname{Red} A$ active power reduction, for load $L$ at bus $i$ in period $t$

$P_{R E D_{-} B(L, t)}$ RedBactive load curtailment, for load $L$ in period $t$

$P_{R E D \_B(L, t)}^{i}$ RedBactive power reduction, for load $L$ at bus $i$ in period $t$

PRED_C(L.t) RedCactive load curtailment, for load $L$ in period $t$

$P_{R E D \_C(L, t)}^{i} \quad$ RedCactive power reduction, for load $L$ at bus $i$ in period $t$

$P_{\text {EAP(DG.t) }} \quad$ Excess available power by DG unit in period $t$, relative to take or pay contracts

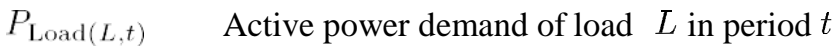

$P_{\text {MaxDR_A(L,t) }}$ Maximum reduction active power, in $\operatorname{Red} A$ program, for load $L$ in period $t$

$P_{\text {Maxdr_B }(L, t)}$ Maximum reduction active power, in RedB program, for load $L$ in period $t$

$P_{\text {Maxdr_C }(L, t)}$ Maximum reduction active power, in $R e d C$ program, for load $L$ in period $t$

$P_{\mathrm{NSD}(L, t)} \quad$ Non-supplied demand for load $L$ in period $t$

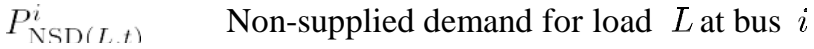
in period $t$

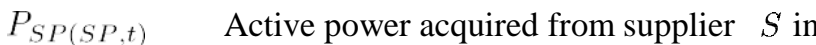
period $t$

$P_{S P(S P, t)}^{i} \quad$ Active power generation of $S P$ external supplier at bus $i$ in period $t$

$P_{\text {SPMax }(S P, t)}$ Maximum active power of $S P$ external supplier in period $t$

$Q_{\mathrm{DG}(\mathrm{DG}, t) \quad \text { Reactive power generation of distributed }}$ generation unit DG in period $t$

$Q_{\mathrm{DG}(\mathrm{DG}, t) \quad \text { Reactive power generation of distributed }}^{i}$ generation unit DG at bus in period $t$

$Q_{\text {DGMax(DG,t) }}$ Maximum reactive power generation of distributed generator unit DG in period $t$

$Q_{\text {DGMin(DG,t) }}$ Minimum reactive power generation of distributed generator unit DG in period $t$

$Q_{\text {Load }(L, t)}^{i} \quad$ Reactive power demand of load $L$ at bus $i$ in period $t$

$Q_{S P(S P, t)} \quad$ Reactive power acquired from supplier $S$ in period $t$

\section{$Q_{S P(S P, t)}^{i} \quad$ Reactive power generation of SP external supplier at bus $i$ in period $t$ \\ $Q_{\text {SPMax }(S P, t)}$ Maximum reactive power of SP external supplier in period $t$ \\ $S_{L k}^{\max }$ \\ Maximum apparent power flow established in line $k$ that connect the bus $i$ and $j$ \\ $T$ \\ $\overline{U_{i(t)}}$ \\ $V_{i(t)}$ \\ $V_{i}^{\max }$ \\ $V_{i}^{\min }$ \\ $X_{\mathrm{DG}(\mathrm{DG}, t)}$ \\ Total number of periods \\ Voltage at bus $i$ in polar form in period $t$ \\ Voltage magnitude at bus $i$ in period $t$ \\ Maximum voltage magnitude at bus $i$ \\ Minimum voltage magnitude at bus $i$ \\ Binary variable of DG unit related to accept the active power generation in period $t$ \\ $\overline{y_{i j}}$ \\ $\overline{y_{S h \_} i}$ \\ Series admittance of line that connect the bus $i$ and $j$ \\ Shunt admittance of line connected in the bus $i$}

\section{INTRODUCTION}

ELECTRIC power systems are not the same anymore.

Be- ginning with the liberalization of electricity markets, several changes have been occurring [1]. The increasingly intensive use of Distributed Generation (DG), the creation of Demand Response (DR) programs [2], [3], and the increasing requirements in terms of energy quality and network reliability have increased the complexity of the infrastructure operation and planning, and aim at bringing to practice the concept of Smart Grid (SG) [4].

In this competitive and complex environment, computational intelligence methods will be required for obtaining solutions for large dimension problems, in an acceptable time [5]. Reference [4] attests the importance of using computational intelligence in many aspects of SG as the system optimization. Particle Swarm Optimization (PSO) is an effective method to determine the solution of large-scale non linear optimization problems [6]. PSO has been successfully applied to several power systems problems [7]-[10].

Aggregation of small-scale distributed resources, as well as their operation, in a competitive environment leads to the creation of Virtual Power Players (VPP) [11]. VPP can aggregate diversity of players and of energy resources, including DR, making them profitable [11].

DR [14], [15] is a very promising resource in the context of electricity markets [12], [13] and SG [14], [16]. A method to determine the DR capacity in a distribution network is presented in [14]. Reference [15] proposes a method to maximize consumers' benefits, regarding their demand reduction in face of the variation in electricity prices and their uncertainties.

In the context of smart grids operated by a virtual power player, the distributed energy resources scheduling gains high relevance due to the competitive environment of smart grid operation. References [17]-[20] report important recent works 
on this field. Those works use different resources optimization points of view: [17], [19], and [20] optimize the grid operation, whereas [18] optimizes the consumer installation. Although network constraints are very relevant in this context, these are usually not included in the proposed optimization models. This inclusion can be done embedding the network simulation in the problem formulation or using a simpler approach, namely the validation of the obtained solutions. The later method is used in [17] which uses a real time digital simulator to validate the solutions. References [19], [20] do not consider network constraints. In this way, the present work is innovative in considering the network constraints in the problem formulation. Moreover, most works that propose a heuristic optimization approach do not assess the quality and the efficiency of the solution by comparing it with the solution obtained with a deterministic approach which is done in the present paper.

This paper deals with the integrated management of demand response and DG in the scope of smart grids. An efficient management of these resources requires the use of adequate methods able to cope with the large-scale non linear optimization problems that result from the large number of distributed energy resources and from the need to ensure that the technical and contractual constraints are not violated. The deterministic approaches are not suitable because their execution times are too high for the operation time constraints. Computational intelligence methods have proved to be able to provide good solutions for the envisaged type of problems in much shorter times.

The present paper proposes a particle swarm optimization based methodology to support the minimization of the operation costs of a virtual power player that manages the resources in a distribution network and the network itself. Those resources include the distributed energy resources available in the considered time period and the energy that can be bought from external energy suppliers. Demand response resources are divided into three capacity programs, namely RedA, RedB, RedC, with distinct price and power characteristics. Network constraints are considered using an AC power flow.

The proposed PSO approach uses Gaussian mutation of the strategic parameters and self-parameterization of the maximum and minimum particle velocities, according to the context. This work is an evolution of the work published in [21]. The previous paper [21] did not consider the network constraints and thus the optimization model used only linear constraints. Additionally, the generation resources are now individually considered whereas in [21] they were aggregated in four distinct types. The proposed PSO approach has been adapted to this new realistic model considering the non linear network constraints. A power flow model [22] has been embedded in the algorithm to enable the analysis of network violations for the swarm solutions. This leads to more accurate solutions than the ones obtained in [21]. The self-parameterization algorithm presented in [21] has been redesigned to deal with the new model.

After this introduction section, Section 2 explains the formulation of the proposed scheduling problem and Section 3 explains the proposed PSO methodology. A case study that considers a real 937-bus distribution network, with 20310 consumers and 548 distributed generators is presented in Section 4. Finally, the most important conclusions of the work are presented in Section 5.

\section{ENERGY RESOURCE SCHEDULINGFORMULATION}

The proposed problem features the minimization of the VPP costs and can be modeled as a mixed-integer non linear optimization problem. The energy resource management requires a multi-period optimization, and the formulation is modeled for a specified time period $C$. This formulation has been implemented in General Algebraic Modeling System (GAMS) [23]. The objective function can be expressed as (1), shown at the bottom of the page.

This objective function leads to the minimization of the costs considering the reduction of load in three different and successive steps (demand response capacity programs RedA, RedB, and RedC), the costs of the energy provided by the external suppliers, and costs of the energy provided by photovoltaic, wind, Combined Heat and Power (CHP), Municipal Solid Waste (MSW), biomass, fuel cell, hydro, and storage. The problem formulation considers the network constraints accounted by an AC power flow.

The constraints of the problem are the following equations (2)-(13):

- The network active (2) and reactive (3) power balance in each period and in each bus . in éach period. During

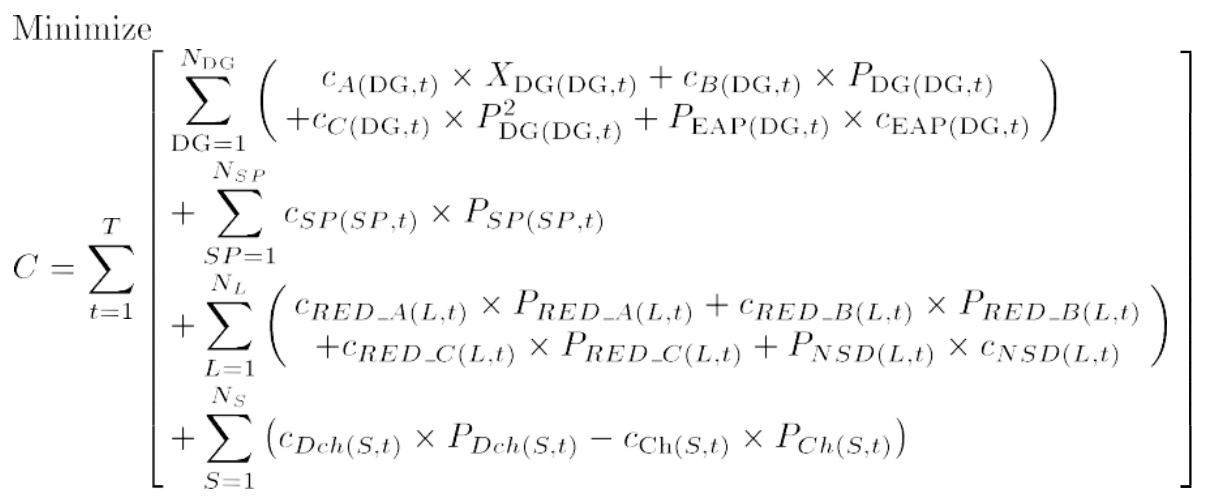


the operation of a power system, both active and reactive power generation must meet the verified demand.

$$
\begin{aligned}
& \sum_{\mathrm{DG}=1}^{N_{\mathrm{DG}}^{i}}\left(P_{\mathrm{DG}(\mathrm{DG}, t)}^{i}-P_{E A P(\mathrm{DG}, t)}^{i}\right) \\
& +\sum_{S P=1}^{N_{S P}^{i}} P_{S P(S P, t)}^{i}+\sum_{S=1}^{N_{S P}^{i}}\left(P_{\mathrm{Dch}(S, t)}^{i}-P_{\mathrm{Ch}(S, t)}^{i}\right) \\
& -\sum_{L=1}^{N_{L}^{i}}\left(P_{\operatorname{Load}(L, t)}^{i}-P_{D R_{-} A(L, t)}^{i}-P_{D R_{-} B(L, t)}^{i}-P_{N S D(L, t)}^{i}\right) \\
& \quad=\sum_{j=1}^{N_{B}} V_{i(t)} \times V_{j(t)}\left(G_{i j} \cos \left(\theta_{i(t)}-\theta_{j(t)}\right)\right. \\
& \left.\quad+B_{i j} \sin \left(\theta_{i(t)}-\theta_{k(t)}\right)\right) \\
& \forall t \in\{1, \ldots, T\} ; \forall i \in\left\{1, \ldots, N_{B}\right\} \quad \text { (2) }
\end{aligned}
$$$$
\sum_{\mathrm{DG}=1}^{N_{\mathrm{DG}}^{i}} Q_{\mathrm{DG}(\mathrm{DG}, t)}^{i}+\sum_{S P=1}^{N_{S P}^{i}} Q_{S P(S P, t)}^{i}-\sum_{L=1}^{N_{L}^{i}} Q_{\mathrm{Load}(L, t)}^{i}
$$$$
=\sum_{j-1}^{N_{B}} V_{i(t)} \times V_{j(t)}\left(G_{i j} \sin \left(\theta_{i(t)}-\theta_{j(t)}\right)\right.
$$$$
\left.-B_{i j} \cos \left(\theta_{i(t)}-\theta_{j(t)}\right)\right)
$$$$
\forall t \in\{1, \ldots, T\} ; \forall i \in\left\{1, \ldots, N_{B}\right\}
$$

- Bus voltage magnitude (4) and angle (5) limits in each bus must be assured. In the reference bus, the voltage magnitude and angle are fixed and set by the user.

$$
\begin{aligned}
& V_{i}^{\min } \leq V_{i(t)} V_{i}^{\max } \\
& \theta_{i}^{\min } \leq \theta_{i(t)} \theta_{i}^{\max } \\
& \forall t \in\{1, \ldots, T\} ; \forall i \in\left\{1, \ldots, N_{B}\right\}
\end{aligned}
$$

- Line thermal limits, which represent the maximum power that can flow in a line due to its characteristics;

$$
\begin{array}{r}
\mid \overline{U_{i(t)}} \times\left[\overline{y_{i j}} \times\left(\overline{U_{i(t)}}-\overline{U_{j(t)}}\right)+\overline{y_{s h-i}} \times \overline{U_{j(t)}}{ }^{*} \mid \leq S_{L k}^{\max }\right. \\
\forall t \in\{1, \ldots, T\} ; \forall i, j \in\left\{1, \ldots, N_{B}\right\} \\
i \neq j ; \forall k \in\left\{1, \ldots, N_{k}\right\} \quad \text { (6) }
\end{array}
$$

- Resources active (7), (9) and reactive (8), (10) power generation limits in each period $t$, respectively for DG units (7), (8) and upstream suppliers (9), (10). The maximum active power that can be reduced in each consumer, in each step, is assured by (11), (12), (13).

$$
\begin{aligned}
& P_{D G M i n(D G, t)} \times X_{D G(D G, t)} \leq P_{D G(D G, t)} \\
& \leq P_{D G M a x(D G, t)} \times X_{D G(D G, t)} \\
& Q_{D G M i n(D G, t)} \times X_{D G(D G, t)} \leq Q_{D G(D G, t)} \\
& \leq Q_{D G M a x(D G, t)} \times X_{D G(D G, t)} \\
& \forall t \in\{1, \ldots, T\} ; \forall D G \in\left\{1, \ldots, N_{D G}\right\} \\
& P_{S P(S P, t)} \leq P_{S P M a x}(S P, t) \\
& Q_{S P(S P, t)} \leq Q_{S P M a x}(S P, t) \\
& \forall t \in\{1, \ldots, T\} ; \forall S P \in\left\{1, \ldots, N_{S P}\right\} \\
& P_{\text {RED } \_A(L, t)} \leq P_{\text {MaxRED } \_A(L, t)}
\end{aligned}
$$

$$
\begin{aligned}
P_{\mathrm{RED} \_} B(L, t) & \leq P_{\text {MaxRed } \_B(L, t)} \\
P_{\mathrm{RED} \_C(L, t)} & \leq P_{\text {MaxRed } \_C}(L, t) \\
\forall t & \in\{1, \ldots, T\} ; \forall L \in\left\{1, \ldots, N_{L}\right\}
\end{aligned}
$$

- Storage technical constraints in each period - The storage units' operation constraints considered in the proposed methodology and implemented in the proposed methods are the ones presented in [24]. Those include multi-period balance of the power in each storage unit as well as the charge and discharge rates.

\section{MutATED PARTICle SWARM APPROACH}

Particle Swarm Optimization (PSO) was created with an initial experiment of a swarm simulator first published in [25]. The authors foresaw its potential for optimization problems, namely to mitigate the difficulties of solving complex problems in terms of computational time. PSO belongs to the category of swarm intelligence methods [26] and computational intelligence. It is used in the present paper to solve the energy resource scheduling problem due to being effective in difficult optimization tasks, namely for non linear large dimension problems [27]. A PSO modified version (PSO-MUT), using Gaussian mutation of the strategic parameters, and self-parameterization is proposed for this purpose. The results and the performance of the proposed method are compared with those obtained with conventional techniques using the professional optimization tool GAMSTM, with the version of PSO without mutation as well as Evolutionary Particle Swarm Optimization (EPSO) found in [28]. The three versions implemented in this paper use a specific modification, namely self-parameterization of the maximum and minimum velocities according to the problem context already introduced in [21]. This will enable a fair comparison of the PSO methodologies in the case study rather than only using the selfparameterization on the proposed PSO-MUT. Self-parameterization was improved from the authors' conference paper [21] to be used in the present ERM problem. Self-parameterization relies on the automatic adjustment of PSO parameters, namely the maximum and the minimum velocities to be set independently from user configuration enabling easy and fast use of the method and also better results. The proposed self-parameterization has been specifically designed for the ERM problem formulation. For other ERM formulations the algorithm rules should be carefully adapted and tested. The maximum and the minimum velocity limits are calculated according to the following: Vector of Prices £ector with generator marginal cost prices and loads demand response cut prices.

For each element (i) of Vector of Prices apply (14) where the variables with lower price will have higher velocities.

$$
\operatorname{maxVel}_{i}=1 /\left(\text { Vector of }_{\text {Prices }}\right)^{1.5}
$$

Fig. 1 depicts the proposed PSO approach for the demand response scheduling problem presented in this paper. The power flow algorithm included in the meta-heuristics is based on the theory given in [22]. The minimum velocity limits for load reduction variables are higher if the highest energy supplier price tends to be expensive compared to the other prices. If the energy 
TABLE I

GENERATOR AND SUPPLIER DATA-EXAMPLE

\begin{tabular}{|c|c|c|c|c|c|}
\hline \multirow{2}{*}{} & \multicolumn{3}{|c|}{ Price Parameters } & \multicolumn{2}{c|}{ Limits } \\
\cline { 2 - 6 } & $\begin{array}{c}\boldsymbol{a} \\
(\boldsymbol{m} \cdot \boldsymbol{u} .)\end{array}$ & $\begin{array}{c}\boldsymbol{b} \\
(\boldsymbol{m} . \boldsymbol{u} \cdot \mathbf{k} \boldsymbol{W})\end{array}$ & $\begin{array}{c}\boldsymbol{c} \\
\left(\boldsymbol{m} \cdot \boldsymbol{u} \cdot \mathbf{k} \boldsymbol{W}^{\mathbf{2}}\right)\end{array}$ & $\begin{array}{c}\text { Lower } \\
(\mathbf{k} \boldsymbol{W})\end{array}$ & $\begin{array}{c}\text { Upper } \\
(\mathbf{k W})\end{array}$ \\
\hline Generator 1 & 0 & 0.20 & 0.001 & 0 & 50 \\
\hline Generator 2 & 0.5 & 0.10 & 0.002 & 0 & 20 \\
\hline Energy supplier 1 & 0 & 0.30 & 0 & 0 & - \\
\hline
\end{tabular}

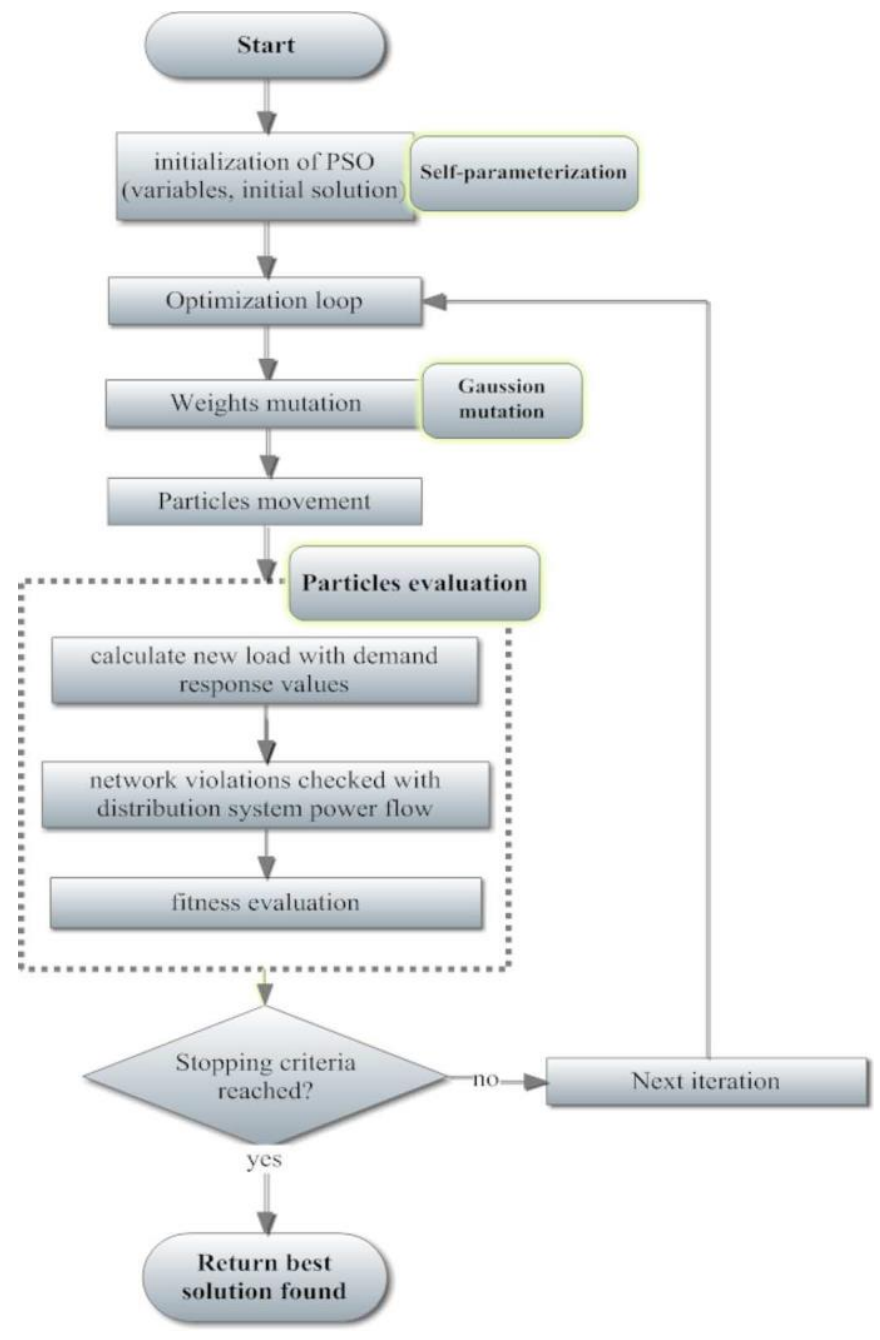

Fig. 1. PSO-MUT for the proposed energy resource scheduling problem.

supplier price tends to be cheaper, then the minimum velocity limits tend to be lower in order to have less load cuts.

The minimum velocity limits are calculated as in (15):

$$
\min V \mathrm{el}=-\frac{\text { Number of variables }}{\text { Position in price rank }}
$$

For better understanding of the above equations, let consider the following example of two generators (Table I) and two loads (Table II). Prices are in monetary units (m.u.).

There are three considered demand response capacity programs with a minimum load reduction for each step. First, the marginal costs of the generators, MC1 for generator 1 (16) and MC2 for generator 2 (17) (shown at the bottom of the page), are calculated using generators' upper limit.

Then, applying (14) for each element of Vector of Prices, we determine maxVel for each of the variables (Table III).

This means that the variable with higher velocity would be the one that corresponds to Generator 2 because it has the lower energy price. On the contrary, the price of RedC of Load1 is the less desired variable to increase given the considered scenario.

The minimum velocity limits of variables are calculated according to the rank position (lowest to highest) of the higher energy supplier contract price, according to (15). In this case there is only one energy supplier contract; it appears in the 7 th place along with RedA of Load1. In this case, considering position 7 and a total of 9 variables, we have:min $\mathrm{Vel}=-9 / 7 \cong$ -1.29 . If this price was the lowest, i.e., the less expensive, therefore being in the 1 st position of the price rank, this would become: $\min V e l=-9 / 1 \cong-9$

In what concerns the mutation of strategic parameters, used only in PSO-MUT, the strategic parameters $(w i)$, are: inertia, memory, and cooperation. The particles movement is ruled by (18).

$$
\begin{aligned}
{ }^{*} v_{i}=w_{i(\text { inertia })} v_{i}+w_{i(\text { memory })}( & \left.b_{i}-x_{i}\right) \\
& +w_{i(\operatorname{cop})}\left(b G-x_{i}\right)
\end{aligned}
$$

The Gaussian mutation is used in each PSO iteration, introducing more diversification in the search process rather than the standard version using fixed and random only weights. At the beginning of the process the values of the corresponding weights are randomly generated between 0 and 1 . After that, the particle's $(i)$ weights (wi) are changed in each iteration of PSO using a Gaussian mutation method according to (19):

$$
{ }^{*} w_{i}=w_{i}+\delta N(0,1)
$$

$$
\begin{aligned}
& M C 1=\frac{0.20 \frac{\mathrm{m} \cdot \mathrm{u}}{\mathrm{kW}} \times 50 \mathrm{~kW}+0.001 \frac{\mathrm{m} \cdot \mathrm{u} .}{\mathrm{kW}^{2}} \times(50 \mathrm{~kW})^{2}}{50 \mathrm{~kW}}=0.25 \mathrm{~m} . \mathrm{u} \cdot / \mathrm{kW} \\
& M C 2=\frac{0.5 \mathrm{~m} \cdot \mathrm{u.}+0.10 \frac{\mathrm{m} \cdot \mathrm{u} \cdot}{\mathrm{kW}} \times 20 \mathrm{~kW}+0.002 \frac{\mathrm{m} \cdot \mathrm{u.}}{\mathrm{kW}} \times(20 \mathrm{~kW})^{2}}{20 \mathrm{~kW}}=0.17 \mathrm{~m} . \mathrm{u} \cdot / \mathrm{kW}
\end{aligned}
$$


TABLE II

Load Curtailment Prices-Example

\begin{tabular}{|c|c|c|c|c|c|}
\hline \multicolumn{2}{|c|}{ Load } & \multicolumn{3}{|c|}{ Load reduction price } & \multirow{2}{*}{$\begin{array}{l}\text { Maximum load } \\
\text { reduction in each } \\
\text { program }(\mathrm{kW})\end{array}$} \\
\hline ID & $\begin{array}{c}\text { Power } \\
(\mathrm{kW})\end{array}$ & $\begin{array}{c}\operatorname{RedA} \\
(\mathrm{m} \cdot \mathrm{u} . / \mathrm{kW})\end{array}$ & $\begin{array}{c}\operatorname{RedB} \\
(\mathrm{m} \cdot \mathrm{u} \cdot / \mathrm{kW})\end{array}$ & $\begin{array}{c}\text { RedC } \\
(\text { m.u. } / \mathrm{kW})\end{array}$ & \\
\hline 1 & 5 & 0.30 & 0.40 & 0.41 & 1 \\
\hline 2 & 25 & 0.20 & 0.35 & 0.40 & 4 \\
\hline
\end{tabular}

TABLE III

MAXimum Velocity Limits of VARIABLES

\begin{tabular}{|l|c|c|c|c|c|c|c|c|c|}
\hline \multirow{2}{*}{ Variables } & \multicolumn{2}{|c|}{ Generators } & \multirow{2}{*}{ Supplier } & \multicolumn{3}{|c|}{ Load1 cut steps } & \multicolumn{3}{|c|}{ Load2 cut steps } \\
\cline { 2 - 8 } & $\mathbf{1}$ & $\mathbf{2}$ & & $\mathbf{A}$ & $\mathbf{B}$ & $\mathbf{C}$ & $\mathbf{A}$ & $\mathbf{B}$ & C \\
\hline $\begin{array}{l}\text { Vector of } \\
\text { Prices }\end{array}$ & 0.25 & 0.17 & 0.30 & 0.30 & 0.40 & 0.41 & 0.20 & 0.35 & 0.40 \\
\hline maxVel & 8.0 & 14.27 & 6.09 & 6.09 & 3.95 & 3.81 & 11.18 & 4.83 & 3.95 \\
\hline
\end{tabular}

where $* w i$ are the resulting particle's weights after mutation, and $\delta$ is the learning parameter, externally fixed between 0 and 1. A high value of $\delta$ gives more importance to mutation. $\mathrm{N}(0,1)$ is a random number following a normal distribution with mean of 0 and a variance of 1 (squared scale). Once again, the strategic parameters are limited to values between 0 and 1 in this stage.

It must be clarified that all the solutions obtained by the PSO based methods use an AC power flow in order to consider the network constraints and the power losses.

\section{CASE Study}

The present case study illustrates the application of the proposed methodology to a set of consumers connected to a real Portuguese distribution network. The considered network is operated by a VPP that aims to minimize the operation costs. VPP operates the existing sources (DG and suppliers) and the demand response capacity programs. In each period, with the aim of facing the existing load demand, the VPP performs the scheduling of the existing resources. Due to the space limitation for presenting results, a single period was considered and the existence of storage units is not taken into account.

Firstly the characterization of the case study scenario is presented in sub-section A. The obtained results with the proposed methodology, with the other PSO approaches and the deterministic approach are then shown and compared in Sub-section B.

\section{A. Scenario Characterization}

The network used in this case study is a real $30 \mathrm{kV}$ distribution network, supplied by one high voltage substation (60/30 $\mathrm{kV}$ ) with 90 MVA of maximum power capacity distributed by 6 feeders, with a total number of 937 buses and 464 MV/LV transformers [24]. Fig. 2 shows the summarized scheme of the distribution network. The number of consumers and the demand in each feeder is also shown.

This distribution network has already been in use for many years and it has suffered many reformulations. It is partly composed of aluminum conductors and partly of copper conductors and the distribution is made by power lines and underground cables. The 20310 consumers connected to this network are classified into five consumer types. The peak power demand is $62630 \mathrm{~kW}$.
Regarding the participation of the consumers in each one of the proposed demand response capacity programs (three successive reduction steps-RedA, RedB, and RedC), a determined power reduction and the respective remuneration price were established for each consumer type. Each reduction step only can be used after the previous one have been fully used (e.g., RedC only can be used when RedA and RedB have been already fully used). For this case study, the maximum power reduction was fixed equal to $10 \%, 5 \%$, and $5 \%$ of each load nominal value, respectively for demand response capacity programs RedA, RedB, and RedC. Table IV presents the values of demand reduction costs in each demand response capacity program. These values correspond to the remuneration values that are paid to the consumers participating in each demand response capacity programs, which are considered fixed for each consumer type.

Table IV also presents the total capacity of demand reduction in each demand response capacity program, regarding the consumer types, for the considered network.

As the original network does not have distributed generation, the energy sources considered for this case study resulted from a study regarding the allocation of DG. Table V presents the values of prices (unitary operation costs), total available capacity, and the number of units for each type of DG technology. The respective values for the ten considered suppliers, connected to the network in the substation, are also presented. Only the linear component of the sources cost functions is considered in this case study.

As it was explained in Section III, the considered optimization has been solved using a deterministic approach and 3 PSO variants approaches (without mutation-PSO - and with mutation-PSO-MUT and EPSO [28]). Table VI presents the values/ description of both PSO methods' parameters. The values for the learning parameter in EPSO were set to 0.8 and the replica was set to 1. Self-parameterization is also introduced in EPSO to enable a closer comparison.

This case study corresponds to a total of 62046 variables. In the PSO methods these variables are coded in memory vectors, i.e., each particle has a dimension space of 62046. The max position of each particle's dimension is defined by the capacity limits of demand response capacity programs and generators/aggregators upper limit power whereas the min position is 


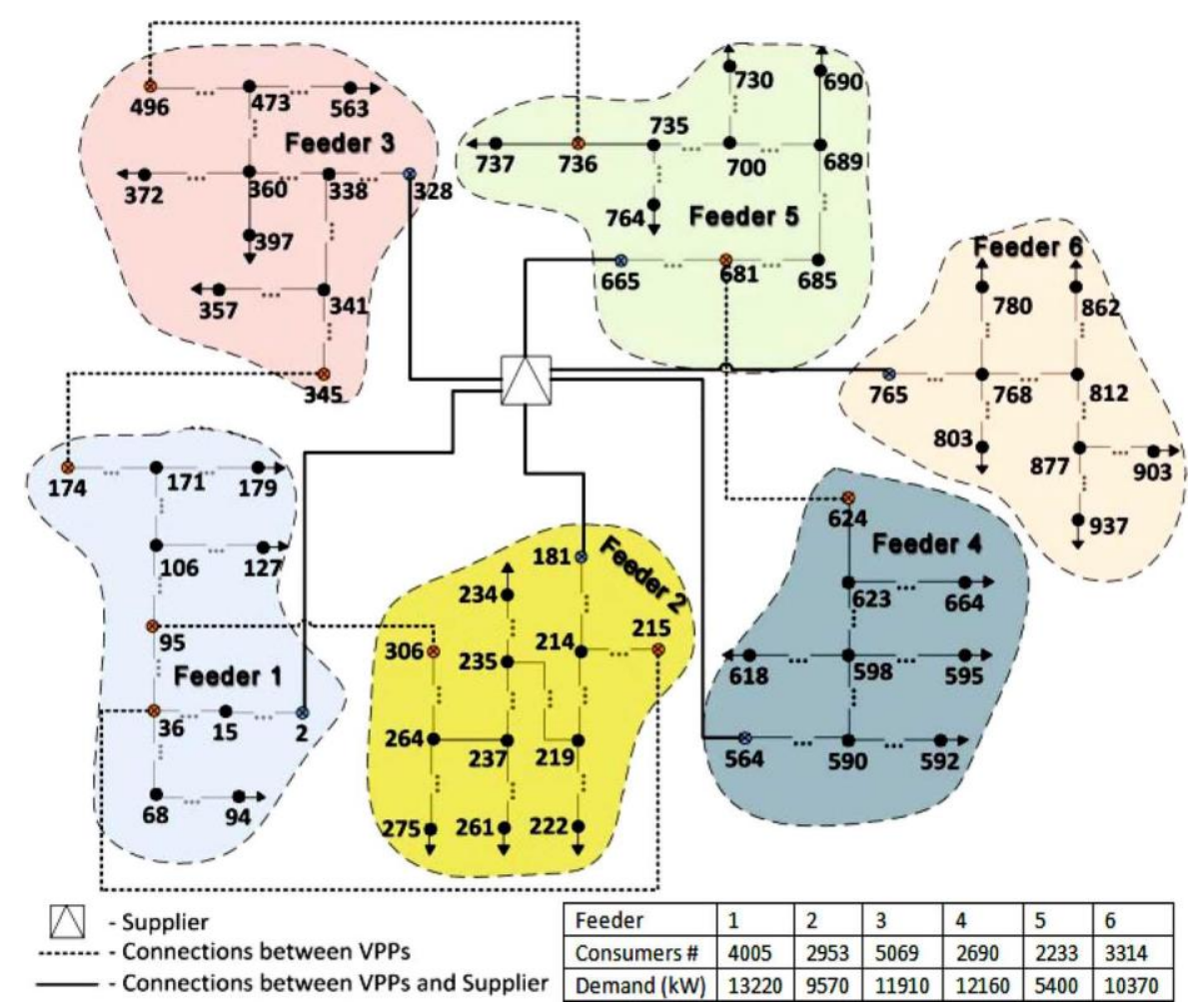

Fig. 2. Distribution network.

TABLE IV

Demand Response Capacity Programs Characterization

\begin{tabular}{l|c|c|c|c|c|c}
\hline \multirow{2}{*}{$\begin{array}{l}\text { Type of } \\
\text { consumer }\end{array}$} & \multicolumn{3}{|l|}{$\begin{array}{l}\text { Reduction capacity } \\
(\mathrm{kW})\end{array}$} & \multicolumn{2}{|c|}{$\begin{array}{l}\text { Reduction costs } \\
(\mathrm{m} . \mathrm{u} . / \mathrm{kWh})\end{array}$} \\
\cline { 2 - 7 } & RedA & RedB & RedC & RedA & RedB & RedC \\
\hline Domestic & 936.9 & 468.47 & 468.47 & 0.16 & 0.20 & 0.24 \\
\hline $\begin{array}{l}\text { Small } \\
\text { Commerce }\end{array}$ & 798.3 & 399.17 & 399.17 & 0.15 & 0.19 & 0.22 \\
\hline $\begin{array}{l}\text { Medium } \\
\text { Commerce }\end{array}$ & 1125.4 & 562.74 & 562.74 & 0.18 & 0.20 & 0.26 \\
\hline $\begin{array}{l}\text { Large } \\
\text { Commerce }\end{array}$ & 1088.0 & 544.02 & 544.02 & 0.17 & 0.24 & 0.26 \\
\hline Industrial & 2314.2 & 1157.1 & 1157.1 & 0.17 & 0.26 & 0.28 \\
\hline Total & 6262.8 & 3131.5 & 3131.5 & - & - & - \\
\hline \hline
\end{tabular}

TABLE V SOURCES

CHARACTERIZATION

\begin{tabular}{l|l|c|c|l|c|c}
\hline Resource & $\begin{array}{c}\text { Price } \\
(\mathrm{m} . \mathrm{u} . \mathrm{kWh})\end{array}$ & $\begin{array}{c}\text { Capacity } \\
(\mathrm{kW})\end{array}$ & $\begin{array}{c}\text { Units } \\
\#\end{array}$ & Resource & $\begin{array}{c}\text { Price } \\
(\mathrm{m} . \mathrm{u} . \mathrm{kWh})\end{array}$ & $\begin{array}{c}\text { Capacity } \\
(\mathrm{kW})\end{array}$ \\
\hline PV & 0.2 & 7061.2 & 208 & Supplier3 & 0.09 & 3000 \\
\hline Wind & 0.05 & 5866.0 & 254 & Supplier4 & 0.11 & 3000 \\
\hline CHP & 0.08 & 6910.1 & 16 & Supplier5 & 0.13 & 3000 \\
\hline Biomass & 0.15 & 2826.5 & 25 & Supplier6 & 0.15 & 3000 \\
\hline MSW & 0.11 & 53.1 & 7 & Supplier7 & 0.17 & 3000 \\
\hline Hydro & 0.15 & 214.0 & 25 & Supplier8 & 0.19 & 3000 \\
\hline Fuel cell & 0.3 & 2457.6 & 13 & Supplier9 & 0.21 & 10000 \\
\hline Supplier1 & 0.05 & 3000.0 & - & Supplier10 & 0.23 & 10000 \\
\hline Supplier2 & 0.07 & 3000.0 & - & Total & - & 69388 \\
\hline \hline
\end{tabular}

zero. This case study uses 20 particles and 150 iterations. These values were obtained by empirical experimentation. Increasing the number of iterations and particles would result in more execution time without significant solution quality gains.
TABLE VI

PARAMETERS OF PSO METHODS

\begin{tabular}{|c|c|c|}
\hline \multirow{2}{*}{ Parameters } & \multicolumn{2}{|c|}{ PSO Methodologies } \\
\hline & PSO & PSO-MUT / EPSO \\
\hline Inertia Weight & 1 & \multirow{3}{*}{$\begin{array}{l}\text { Gaussian mutation } \\
\text { weights }\end{array}$} \\
\hline $\begin{array}{l}\text { Acceleration Coefficient } \\
\text { Best Position }\end{array}$ & 2 & \\
\hline Cooperation Coefficient & 2 & \\
\hline Initial swarm population & \multicolumn{2}{|c|}{$\begin{array}{l}\text { Randomly generated between the upper and } \\
\text { lower bounds of the variables }\end{array}$} \\
\hline Stopping Criteria & \multicolumn{2}{|c|}{150 iterations } \\
\hline Max. velocity & \multicolumn{2}{|c|}{ Refer to Section III } \\
\hline Min. velocity & \multicolumn{2}{|c|}{ Refer to Section III } \\
\hline
\end{tabular}

\section{B. Results}

This sub-section presents the results obtained for this case study energy resources management. In order to evaluate the effectiveness of the PSO methods and of the proposed energy resources management methodology, the results are shown regarding the three approaches (deterministic mixed integer non linear programming optimization obtained in GAMS; heuristic optimization obtained the proposed PSO, labeled as "PSO"; and heuristic optimization obtained by the proposed mutated version of PSO, labeled as "PSO-MUT"). The number of particles assigned to PSO and PSO-MUT methods was 20. The number of iterations was 150 for both versions in order to allow comparing the results. The details of the results shown in Figs. 3 to 6 correspond to a random solution obtained by the PSO and by the PSO-MUT.

Fig. 3 shows the results of the resource scheduling. The solutions obtained by the deterministic approach (labeled as "GAMS") are considered as the reference. It is possible to see 


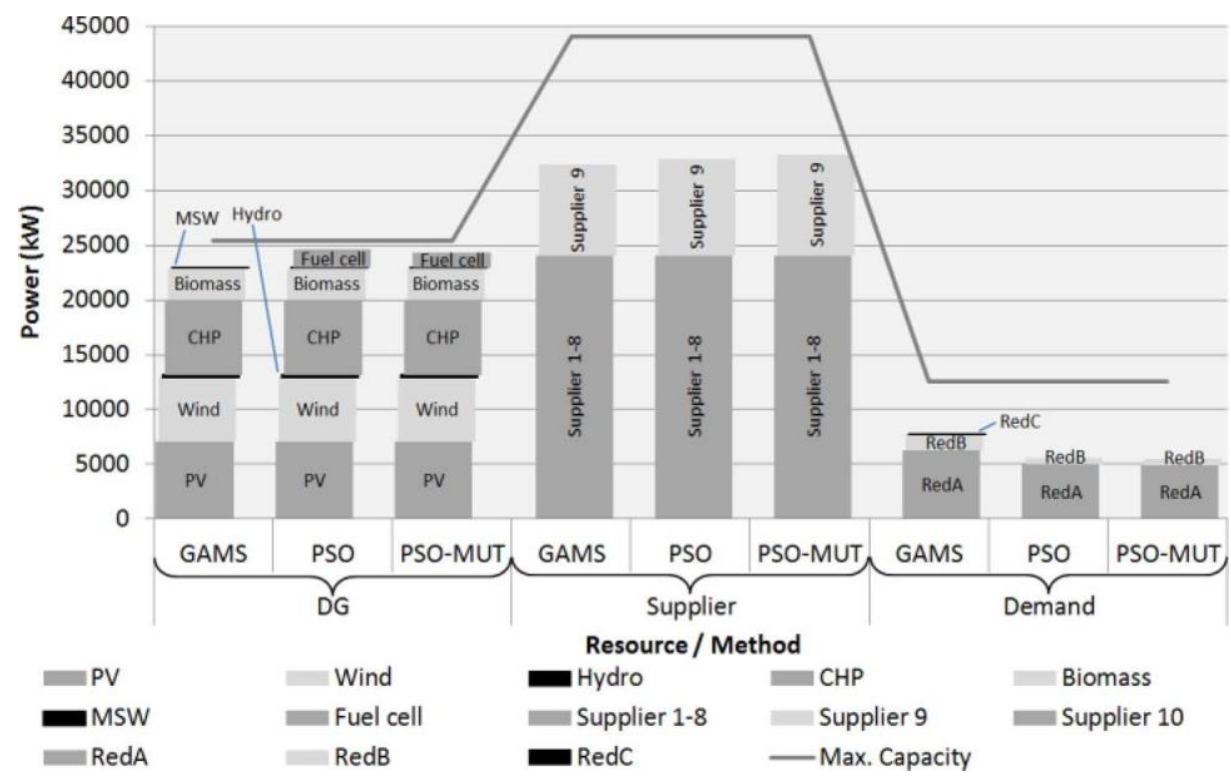

Fig. 3. Energy resource scheduling.

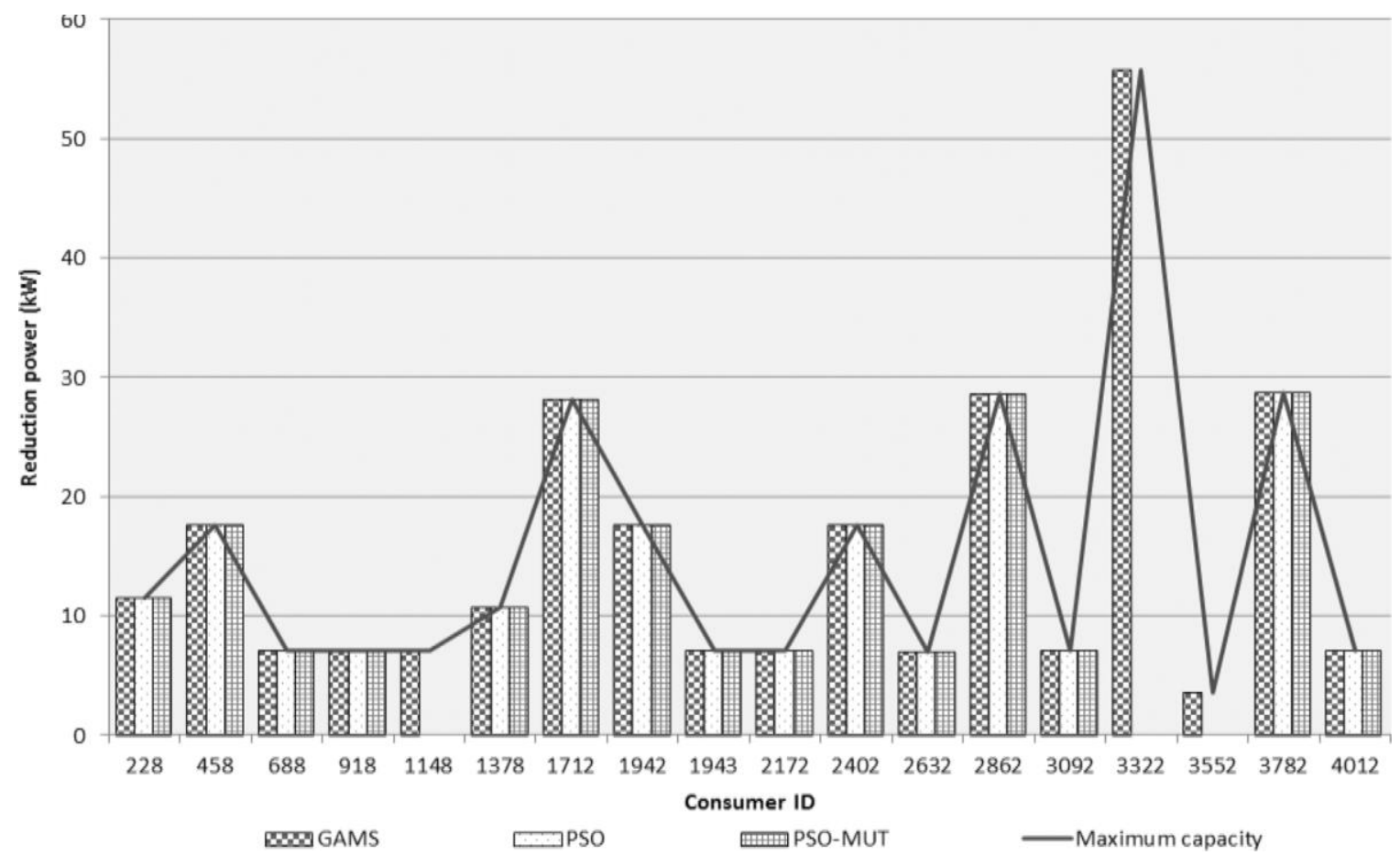

Fig. 4. Feeder 1 medium commerce consumers schedule in the RedA program.

that the PSO methods schedule all the resources (but not all their available capacity), while the deterministic approach does not make use of fuel cell units due to their operation costs. On contrary, demand response capacity programs are more used in the solution obtained by GAMS. The energy provided by the suppliers is more used by the solution obtained by PSO-MUT.

The proposed methodology schedules each resource individually. However, here the results are presented in a condensed form, showing the total aggregated amount of power scheduled for each type of DG technology, each type of consumer, etc. Fig. 4 shows more detailed results for the medium com- merce consumers in feeder 1 in what concerns the schedule in the RedA demand response capacity program.

For these consumers, the deterministic approach schedules all the consumers for participation in the demand reduction program whereas the solutions obtained by the PSO methods do not scheduled some of the consumers for participation.

Fig. 5 presents the solutions costs obtained for each type of resource. Three components of the objective function are represented, namely DG, supplier and demand response costs. The costs of demand response capacity programs are calculated according to the consumers scheduled by each method. As ex- 


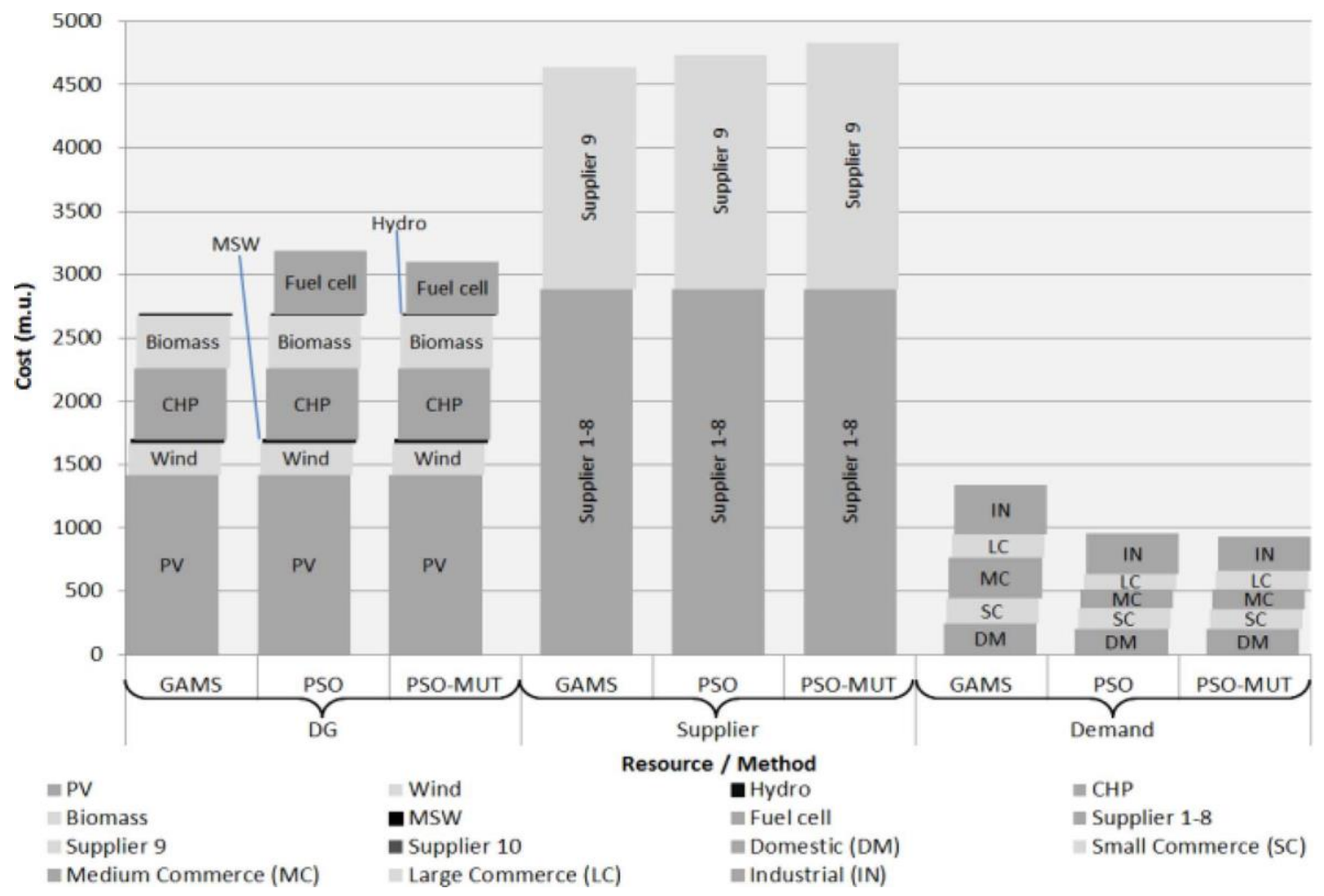

Fig. 5. Energy resource scheduling costs.

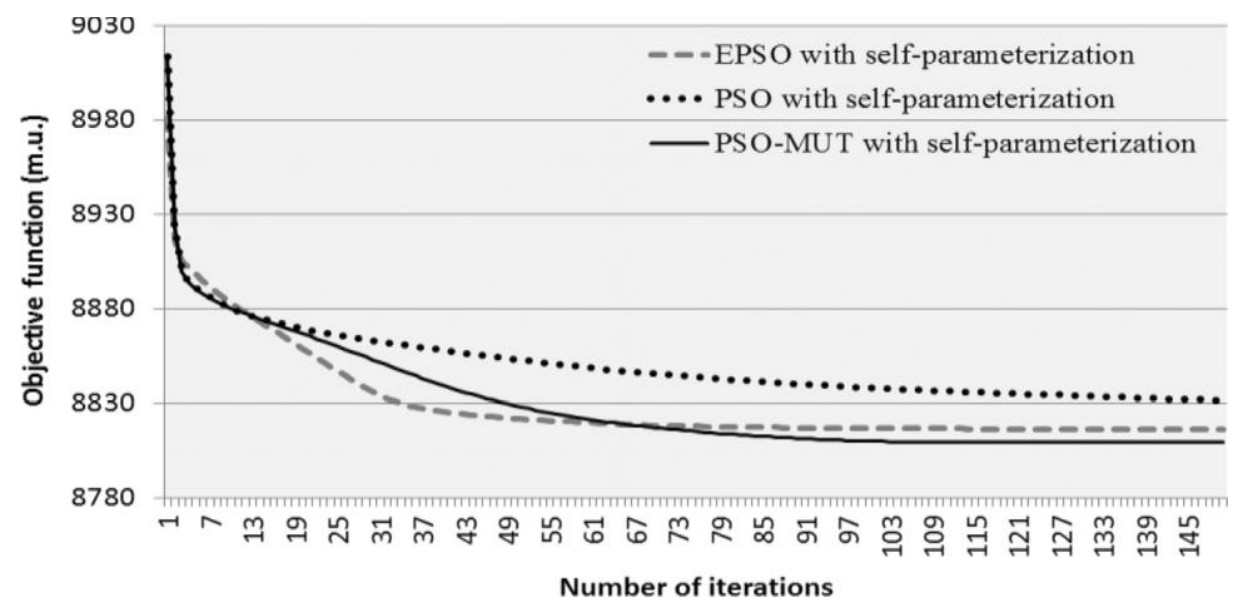

Fig. 6. Evolution of the average solutions of the PSO methods.

pected, the differences between the solutions obtained by the three approaches are related to the schedules presented in Fig. 3.

Fig. 6 shows the average solution found in each iteration for 1000 runs of the three considered PSO based approaches. It is clear that the proposed PSO-MUT has a faster evolution to the solution, which is also the best found solution among the PSO methods.

Table VII shows the values of both operation costs and execution time for the four considered approaches.

These results show that the three PSO-based methods are able to obtain solutions very close to the value obtained with the deterministic approach (8662.6 m.u.) in much faster execution time. The proposed PSO-MUT leads to the best average value ( 8809.2 m.u.) with the minimum standard deviation. The proposed PSO-MUT execution time is slightly higher than the one of the PSO without mutation and significantly lower than the EPSO execution time. The EPSO execution time is higher mainly due to the replication of the swarm and to the required evaluations of the swarm and replica solutions.

\section{CONCLUSION}

The future context of operation of distribution networks, according to the smart grid paradigm, will accommodate large amounts of distributed generation. Enhancement of service quality, increase of energy efficiency and reduction of the operation costs are expected in the new context. Virtual power players will play an important role managing and operating 
TABLE VII

EXeCUTION Time AND OPERATION COSTS VALUeS COMPARISON

\begin{tabular}{l|c|c|c|c|c|c|c|c|c}
\hline \multirow{2}{*}{ Method } & \multicolumn{2}{|c|}{$\begin{array}{c}\text { Execution } \\
\text { time }\end{array}$} & \multicolumn{7}{c}{ Objective function } \\
\cline { 2 - 10 } & \multirow{2}{*}{$(\mathrm{s})$} & \multirow{2}{*}{$(\%)$} & \multicolumn{2}{|c|}{ Best } & \multicolumn{2}{c}{ Worst } & \multicolumn{2}{|c}{ Average } & $\begin{array}{c}\text { Standard } \\
\text { deviation }\end{array}$ \\
\cline { 3 - 10 } & & & (m.u.) & $(\%)$ & (m.u.) & $(\%)$ & (m.u.) & $(\%)$ & (m.u.) \\
\hline GAMS & 1510 & 100 & 8662.6 & 100 & - & - & - & - & - \\
\hline PSO & 59 & 3.9 & 8768.2 & 101.1 & 8876.6 & 102.5 & 8831.3 & 101.9 & 24.8 \\
\hline EPSO & 127 & 8.4 & 8745.1 & 101.0 & 8870.8 & 102.4 & 8816.1 & 101.8 & 29.3 \\
\hline $\begin{array}{l}\text { PSO- } \\
\text { MUT }\end{array}$ & 68 & 4.5 & 8726.2 & 100.7 & 8876.9 & 102.5 & 8809.2 & 101.7 & 22.5 \\
\hline \hline
\end{tabular}

aggregated energy resources, which also include demand response. Due to the competitive environment, important decisions must be as efficient as possible and be taken in short time horizons; computational intelligence methods will be very important in this field.

In this paper, a Particle Swarm Optimization (PSO) based methodology is proposed and applied to the schedule of several energy resources, including demand response, distributed generation, and the energy that can be bought to a set of suppliers, minimizing the operation costs from the point of view of a VPP that operates the network. The proposed method uses Gaussian mutation of the strategic parameters and self-parameterization of the maximum and minimum particle velocities, according to the context.

The application of the proposed method has been illustrated with a case study based on a real 937 bus distribution network, with 20310 consumers and 548 distributed generators. The solutions obtained with the proposed method are compared with those obtained with a deterministic approach, the classic PSO, and the Evolutionary PSO (EPSO). The proposed PSO-MUT approach leads to the best average solution and presents execution times only slightly higher than traditional PSO.

\section{REFERENCES}

[1] D. Kirschen, "Demand-side view of electricity markets," IEEETrans. Power Syst., vol. 18, no. 2, pp. 520-527, May 2003.

[2] C. Woo and L. Greening, "Special issue demand response resources: The US and international experience introduction," Energy, vol. 35, pp. 1515-1517, Apr. 2010.

[3] J. Bushnell, B. Hobbs, and F. Wolak, "When it comes to demand response, is FERC its own worst enemy?," The Electricity J., vol. 22, no. 8, pp. 9-18, Oct. 2009.

[4] G. K. Venayagamoorthy, "Dynamic, stochastic, computational, and scalable technologies for smart grids," IEEE Comput. Intell. Mag., vol. 6, no. 3, pp. 22-35, Aug. 2011.

[5] H. He and A. Kusiak, "Special issue on computational intelligence in smart grid [Guest Editorial]," IEEE Comput. Intell. Mag., vol. 6, no. 3, pp. 12-64, Aug. 2011.

[6] Y. del Valle, G. K. Venayagamoorthy, S. Mohagheghi, J. Hernandez, and R. Harley, "Particle swarm optimization: Basic concepts, variants and applications in power systems," IEEE Trans. Evol. Comput., vol. 12, no. 2, pp. 171-195, Apr. 2008.

[7] M. R. AlRashidi and M. E. El-Hawary, "A survey of particle swarm optimization applications in electric power systems," IEEE Trans. Evol. Comput., vol. 13, pp. 913-918, Aug. 2009.

[8] K. Y. Lee and M. A. El-Sharkawi, Modern Heuristic Optimization Techniques: Theory and Applications to Power Systems. Hoboken, NJ: IEEE Press/Wiley-Interscience, 2008.
[9] Y. Yare, G. Venayagamoorthy, and U. Aliyu, "Optimal generator maintenance scheduling using a modified discrete PSO," in IET Proc. Generation, Transmission and Distribution, Nov. 2008, vol. 2, no. 6, pp. 834-846.

[10] B. Luitel and G. K. Venayagamoorthy, "Particle swarm optimization with quantum infusion for system identification," Engineering Applications of Artificial Intelligence, vol. 23, no. 5, pp. 635-649, Aug. 2010.

[11] T. Pinto, Z. A. Vale, H. Morais, I. Praça, and C. Ramos, "Multi-agent based electricity market simulator with VPP: Conceptual and implementation issues," in Proc. IEEE Power \& Energy Society General Meeting, 2009, Jul. 26-30, 2009, pp. 1-9.

[12] P. Faria, Z. Vale, J. Soares, and J. Ferreira, "Demand response management in power systems using a particle swarm optimization approach," IEEE Intell. Syst., 2011, DOI: 10.1109/MIS.2011.35.

[13] P. Faria and Z. Vale, "Demand response in electrical energy supply: An optimal real time pricing approach," Energy, vol. 36, no. 8, pp. 5374-5384, Aug. 2011.

[14] J. Medina, N. Muller, and I. Roytelman, "Demand response and distribution grid operations: Opportunities and challenges," IEEE Trans. Smart Grid, vol. 1, no. 2, pp. 193-198, Sep. 2010.

[15] A. J. Conejo, J. M. Morales, and L. Baringo, "Real-time demand response model," IEEE Trans. Smart Grid, vol. 1, no. 3, pp. 236-242, Dec. 2010

[16] Z. Vale, T. Pinto, H. Morais, I. Praca, and P. Faria, "VPP's multi-level negotiation in smart grids and competitive electricity markets," in Proc. IEEE Power and Energy Society General Meeting, Jul. 24-29, 2011, pp. 1-8.

[17] T. Logenthiran, D. Srinivasan, A. M. Khambadkone, and H. N. Aung, "Multiagent system for real-time operation of a microgrid in real-time digital simulator," IEEE Trans. Smart Grid, vol. 3, no. 2, pp. 925-933, Jun. 2012.

[18] M. Pedrasa, T. Spooner, and I. MacGill, "Coordinated scheduling of residential distributed energy resources to optimize smart home energy services," IEEE Trans. Smart Grid, vol. 1, no. 2, pp. 134-143, Sep. 2010.

[19] A. Saber and G. Venayagamoorthy, "Efficient utilization of renewable energy sources by gridable vehicles in cyber-physical energy systems," IEEE Syst. J., vol. 4, no. 3, pp. 285-294, Sep. 2010.

[20] M. Hopkins, A. Pahwa, and T. Easton, "Intelligent dispatch for distributed renewable resources," IEEE Trans. Smart Grid, vol. 3, no. 2, pp. 1047-1054, Jun. 2012.

[21] P. Faria, Z. Vale, J. Soares, and J. Ferreira, "Particle swarm optimization applied to integrated demand response resources scheduling," in Proc. IEEE Symp. Computational Intelligence Applications in Smart Grid (CIASG), Apr. 11-15, 2011, pp. 1-8.

[22] D. Thukaram, H. M. W. Banda, and J. Jerome, "A robust three phase power flow algorithm for radial distribution systems," Electrical Power Syst. Res., vol. 50, pp. 227-236, 1999.

[23] GAMS-The Solver Manuals. GAMS Development Corp., Washington, DC, 2007.

[24] S. Gonçalves, H. Morais, T. Sousa, and Z. Vale, "Energy resource scheduling in a real distribution network managed by several virtual power players," presented at the 2012 IEEE Power and Energy Society Transmission and Distribution Conf., Orlando, FL, 2012.

[25] J. Kennedy and R. C. Eberhart, "Particle swarm optimization," in Proc. IEEE Int. Conf. Neural Networks, Dec. 1995, vol. 4, pp. 1942-1948. 
[26] E. Bonabeau, M. Dorigo, and G. Theraulaz, Swarm Intelligence: From Natural to Artificial Systems. Oxford, U.K.: Oxford Univ. Press, 1999.

[27] A. Engelbrecht, Computational Intelligence: An Introduction. New York: Wiley, 2007.

[28] V. Miranda, "Evolutionary algorithms with particle swarm movements," in Proc. 13th Int. Conf. Intelligent Systems Application to Power Systems, Nov. 6-10, 2005, pp. 6-21. 